\title{
The product of two Dirichlet series
}

\author{
by
}

FrÉdÉRIC BAYART (Lille)

1. Introduction. Let $A(s)=\sum_{n \geq 1} a_{n} n^{-s}$ be a Dirichlet series. Two abscissas are connected to the convergence of $A$ :

$\sigma_{\mathrm{c}}=\inf \left\{\sigma \in \mathbb{R} ; \sum_{n=1}^{+\infty} a_{n} n^{-\sigma}\right.$ converges $\}, \quad$ abscissa of convergence,

$\sigma_{\mathrm{a}}=\inf \left\{\sigma \in \mathbb{R} ; \sum_{n=1}^{+\infty}\left|a_{n}\right| n^{-\sigma}\right.$ converges $\}$, abscissa of absolute convergence.

It is well known that $0 \leq \sigma_{\mathrm{a}}-\sigma_{\mathrm{c}} \leq 1$, and that those inequalities are best possible. We recall that $A$ converges for $\operatorname{Re}(s)>\sigma_{\mathrm{c}}$, and $A$ diverges for $\operatorname{Re}(s)<\sigma_{\mathrm{c}}$. If $\sigma_{\mathrm{c}}>0$, we have moreover the following Hadamard-like formula:

$$
\sigma_{\mathrm{c}}=\limsup _{n \rightarrow+\infty} \frac{\log \left|A_{n}\right|}{\log n}
$$

where $A_{n}=a_{1}+\ldots+a_{n}$.

Let $B(s)=\sum_{n>1} b_{n} n^{-s}$ be another Dirichlet series. The Dirichlet product $C=A B$ is formally defined by $C(s)=\sum_{n \geq 1} c_{n} n^{-s}$, where $c_{n}=$ $\sum_{i j=n} a_{i} b_{j}$. It is natural to study the relations between the abscissas of convergence of $A, B$ and $C$. The answer is given by the following:

Theorem 1.1. If $A$ (resp. B) is a Dirichlet series whose abscissa of convergence is $\alpha$ (resp. $\beta$ ), with $|\alpha-\beta| \leq 1$, then the abscissa of convergence of $C=A B$ is less than $\frac{1}{2}(\alpha+\beta+1)$. Moreover, this inequality is optimal: we can find $A$ and $B$ such that $C$ diverges for all $\sigma<\frac{1}{2}(\alpha+\beta+1)$.

This theorem has a long history. Its first part was first proved by Stieltjes if $\alpha=\beta=0$, next by Landau in the general case. Moreover, Landau [4] proved that $\sigma_{\mathrm{c}}$ can be larger than $\frac{1}{2}(\alpha+\beta)+\frac{1}{8}$, whereas it had been conjectured by Cahen that we always have $\sigma_{\mathrm{c}} \leq \frac{1}{2}(\alpha+\beta)$. It is Bohr [1] who gave the first proof of the optimality of the bound $\frac{1}{2}(\alpha+\beta+1)$. His method,

2000 Mathematics Subject Classification: Primary 11M41. 
which used the order function, was simplified by H. Queffélec in [5], and generalized by J. P. Kahane and H. Queffélec in [2], by using the Baire category theorem. Very recently, S. V. Konyagin and H. Queffélec have given in [3] an easy proof of this theorem, by using the principle of uniform boundedness.

The previous arguments are essentially topological. In Section 2, we give the first "explicit" proof of the optimality. Indeed, we give an example of two Dirichlet series $A$ and $B$ with $\sigma_{\mathrm{c}}(A)=\alpha, \sigma_{\mathrm{c}}(B)=\beta$, and $\sigma_{\mathrm{c}}(A B)=$ $\frac{1}{2}(\alpha+\beta+1)$.

In Section 3, we study the same problem under additional assumptions on $A$ and $B$. For instance, $B$ will be the $\zeta$ function, and $A$ will satisfy $A(1)=0$. Under these conditions, we show that $\sigma_{\mathrm{c}}(A B) \leq \frac{1}{2}(\alpha+\beta)$, the inequality being optimal. This answers a question asked by M. Balazard.

2. Convergence of products of Dirichlet series. We begin by the following simple

Lemma 2.1. Let $N$ be the square of an even number. For $k=1, \ldots, \sqrt{N} / 2$, set $i_{k}=[N / k]$. Then:

- $\left[N / i_{k}\right]=k$.

- $2 \sqrt{N} \leq i_{k} \leq N$.

We recall that $[x]$ denotes the integer part of $x$.

Proof. Clearly, we have

$$
2 \sqrt{N}=\left[\frac{N}{\sqrt{N} / 2}\right] \leq i_{k} \leq N=\left[\frac{N}{1}\right] .
$$

Moreover, since $i_{k}=[N / k]$, we see that $N / i_{k} \geq k$ and $N / i_{k}<N k /(N-k)$. The assumption $k \leq \sqrt{N} / 2$ allows us to conclude.

Now, we are going to define two Dirichlet series $A$ and $B$ with $\sigma_{\mathrm{c}}(A)=\alpha$, $\sigma_{\mathrm{c}}(B)=\beta$, and $\sigma_{\mathrm{c}}(A B)=\frac{1}{2}(\alpha+\beta+1)$, with the additional assumption $\beta-1<\alpha<\beta$. By a translation, it is sufficient to handle the case $\beta=1$. For all $n \geq 1$, we set $M_{n}=2^{4^{n}}$, so that $M_{n-1}=M_{n}^{1 / 4}$. Lemma 2.1 gives us integers $i_{k, n}$ which satisfy

$$
2 \sqrt{M_{n}} \leq i_{\sqrt{M_{n}} / 2, n}<\ldots<i_{2, n}<i_{1, n} \leq M_{n}
$$

and

$$
\left[\frac{M_{n}}{i_{k, n}}\right]=k \quad \text { for } k=1, \ldots, \sqrt{M_{n}} / 2 .
$$

If $n$ is fixed, the integers $i_{k, n}$ are all distinct. Moreover, since $M_{n-1}<2 \sqrt{M_{n}}$, if $(k, n) \neq(j, m)$, then $i_{k, n} \neq i_{j, m}$. So we may define a sequence $\left(a_{i}\right)_{i \in \mathbb{N}}$ by

$$
a_{i_{k, n}}=(-1)^{k} i_{k, n}^{\alpha}, \quad a_{i}=0 \quad \text { if } i \neq i_{k, n} .
$$


The Dirichlet series $A(s)=\sum_{i>1} a_{i} i^{-s}$ is then well defined. Let us compute its abscissa of convergence. If $N \geq 1$, and if $n_{0}$ is the least integer such that $N \leq M_{n_{0}}$, then

$$
\sum_{i=1}^{N} a_{i} i^{-\alpha}=\sum_{n=1}^{n_{0}-1} \sum_{k=1}^{\sqrt{M_{n}} / 2}(-1)^{k}+\sum_{i_{k, n_{0}} \geq 2 \sqrt{M_{n_{0}}}}^{N}(-1)^{k} .
$$

But for all $n \geq 1, \sqrt{M_{n}} / 2$ is an even integer, and so

$$
\sum_{k=1}^{\sqrt{M_{n}} / 2}(-1)^{k}=0
$$

Since

$$
\sum_{i_{k, n_{0}} \geq 2 \sqrt{M_{n_{0}}}}^{N}(-1)^{k} \in\{-1,0,1\},
$$

the abscissa of convergence of $A$ is $\alpha$.

We define $B$ as the alternate zeta function, namely

$$
B(s)=\sum_{i \geq 1}(-1)^{i} i^{-s} .
$$

Clearly, $\sigma_{\mathrm{c}}(B)=0$. As a consequence of Theorem 1.1, we get $\sigma_{\mathrm{c}}(A B) \leq$ $\frac{1}{2}(\alpha+1)$. In fact, we have equality:

TheOREM 2.2. The abscissa of convergence of $A B$ is exactly $\frac{1}{2}(1+\alpha)$.

Proof. We set $C(s)=A(s) B(s)=\sum_{n \geq 1} c_{n} n^{-s}$, and $C_{N}=c_{1}+\ldots+c_{N}$. An elementary computation gives

$$
C_{N}=\sum_{i=1}^{N} a_{i} B_{[N / i]},
$$

where $B_{n}=b_{1}+\ldots+b_{n}$. We suppose that $N=M_{n}$. It is sufficient to prove that $C_{N} \geq \delta N^{(\alpha+1) / 2}$. Observe that

$$
B_{j}= \begin{cases}-1 & \text { if } j \text { is odd } \\ 0 & \text { if } j \text { is even. }\end{cases}
$$

We split the sum which defines $C_{N}$ into two parts:

$$
C_{N}=\sum_{i=1}^{2 \sqrt{N}-1} a_{i} B_{[N / i]}+\sum_{i=2 \sqrt{N}}^{N} a_{i} B_{[N / i]}=: S_{1}+S_{2} .
$$

If $i \leq 2 \sqrt{N}-1$, the condition $a_{i} \neq 0$ implies that $i \leq M_{n-1}=N^{1 / 4}$. In 
particular,

$$
\left|S_{1}\right| \leq \sum_{i=1}^{N^{1 / 4}}\left|a_{i}\right| \leq N^{1 / 4} N^{\alpha / 4}
$$

On the other hand,

$$
S_{2}=\sum_{k=1}^{\sqrt{N} / 2} a_{i_{k, n}} B_{k}=\sum_{\substack{k=1 \\ k \text { odd }}}^{\sqrt{N} / 2}(-1) \cdot(-1) i_{k, n}^{\alpha} .
$$

Since $i_{k, n} \geq 2 \sqrt{N}$, we get $S_{2} \geq K_{1} \sqrt{N} N^{\alpha / 2}$. Therefore $C_{N} \geq K_{2} N^{(1+\alpha) / 2}$, which shows that the abscissa of convergence of $C$ is at least $(1+\alpha) / 2$.

Remark 2.3. The case $\alpha \leq \beta-1$ is easier. Indeed, the inequality $\sigma_{\mathrm{a}}(A)$ $\leq \sigma_{\mathrm{c}}(A)+1$ implies $\sigma_{\mathrm{c}}(A B) \leq \beta$. This is optimal. For instance, set $A(s)=$ $\sum_{n \geq 1}(-1)^{n} n^{\alpha} n^{-s}, \sigma_{\mathrm{c}}(A)=\alpha$, and $B(s)=\sum_{n \geq 1}(-1)^{n} n^{\beta} n^{-s}, \sigma_{\mathrm{c}}(B)=\beta$. If $p$ is a prime number, one has

$$
c_{p}=a_{p} b_{1}+a_{1} b_{p}=(-1)^{p}\left(p^{\alpha}+p^{\beta}\right),
$$

so that $\left|c_{p}\right| \geq K_{3} p^{\beta}$. In particular, this gives $\sigma_{\mathrm{c}}(A B) \geq \beta$.

3. Multiplication by $\zeta$. We would like to know if the abscissa of convergence of the product $A B$ can be improved if $A$ vanishes at least once on the half-line $] \alpha,+\infty[$. M. Balazard noticed (personal communication) that if $0<\sigma_{\mathrm{c}}(A)<1$ and $A(1)=0$, then the abscissa of convergence of the product $A \zeta$ is less than $\frac{1}{2}(\alpha+1)$. He asked whether this inequality is optimal.

Our aim in this section is to generalize (in somewhat optimal form) Balazard's observation, and to answer his question.

Theorem 3.1. Let $A(s)=\sum_{n \geq 1} a_{n} n^{-s}$ and $B(s)=\sum_{n \geq 1} b_{n} n^{-s}$ be two Dirichlet series with $\sigma_{\mathrm{c}}(A)=\alpha, \sigma_{\mathrm{c}}(B)=\beta$, and $\beta-1<\alpha<\beta$. Moreover, suppose that

1. $A(\beta)=0$,

2. $B_{n}=b_{1}+\ldots+b_{n}=K n^{\beta}+O\left(n^{\beta-1}\right)$.

Then the abscissa of convergence of the product $A B$ is less than $\frac{1}{2}(\alpha+\beta)$.

This theorem improves the abscissa given by Theorem 1.1, since we gain a translation of factor $1 / 2$. In particular, if $B=\zeta$, we have $B_{n}=n$, and we recover Balazard's observation.

Proof. We can assume that $K=\beta=1$. We set $C(s)=\sum_{n=1}^{+\infty} c_{n} n^{-s}=$ $A(s) B(s)$; it is sufficient to prove that $C_{n}=c_{1}+\ldots+c_{n}=O\left(n^{(1+\alpha) / 2+\varepsilon}\right)$ for each $\varepsilon>0$. We shall use the hyperbola method of Dirichlet by writing 
(see $[6$, p. 38])

$$
C_{n}=\sum_{i \leq \sqrt{n}} a_{i} B_{[n / i]}+\sum_{j \leq \sqrt{n}} b_{j} A_{[n / i]}-A_{[\sqrt{n}]} B_{[\sqrt{n}]}=: S_{1}+S_{2}-S_{3} .
$$

Now, we know that:

(i) $\left|\sum_{n \leq t} a_{n}\right|=O\left(t^{\alpha+\varepsilon}\right)$,

(ii) $\sigma_{\mathrm{a}}(A) \leq \alpha+1$, and so $\sum_{n \leq t}\left|a_{n}\right|=O\left(t^{1+\alpha+\varepsilon}\right)$.

Therefore, we have

$$
S_{3}=O\left(\sqrt{n} n^{\alpha / 2+\varepsilon}\right)=O\left(n^{(1+\alpha) / 2+\varepsilon}\right), \quad S_{2}=\sum_{j \leq \sqrt{n}} b_{j} O\left(\frac{n^{\alpha+\varepsilon}}{j^{\alpha+\varepsilon}}\right) .
$$

But $b_{j}=B_{j}-B_{j-1}=j-(j-1)+O(1)=O(1)$, and so

$$
S_{2}=\sum_{j \leq \sqrt{n}} O\left(\frac{n^{\alpha+\varepsilon}}{j^{\alpha+\varepsilon}}\right)=O\left(n^{(1+\alpha) / 2+\varepsilon}\right) .
$$

Taking advantage of $A(1)=0$, we have

$$
S_{1}=\sum_{i \leq \sqrt{n}} a_{i} B_{[n / i]}-n \sum_{i=1}^{+\infty} \frac{a_{i}}{i}=\sum_{i \leq \sqrt{n}} a_{i}\left(B_{[n / i]}-\frac{n}{i}\right)-n \sum_{i>\sqrt{n}} \frac{a_{i}}{i} .
$$

Now, $B_{[n / i]}-n / i=B_{[n / i]}-[n / i]+[n / i]-n / i=O(1)$, and so

$$
\sum_{i \leq \sqrt{n}} a_{i}\left(B_{[n / i]}-\frac{n}{i}\right)=O\left(\sum_{i \leq \sqrt{n}}\left|a_{i}\right|\right)=O\left(n^{(1+\alpha) / 2+\varepsilon}\right) .
$$

Finally, an Abel summation by parts shows that

$$
n \sum_{i>\sqrt{n}} \frac{a_{i}}{i}=O\left(n^{(1+\alpha) / 2+\varepsilon}\right) .
$$

Putting this together, we find $C_{n}=O\left(n^{(1+\alpha) / 2+\varepsilon}\right)$, which is the conclusion of Theorem 3.1.

We shall prove that Theorem 3.1 is optimal, thus answering the question of M. Balazard.

THEOREM 3.2. Let $\alpha, \beta$ be real numbers such that $\alpha<\beta<\alpha+1$. For each Dirichlet series $B(s)=\sum_{n>1} b_{n} n^{-s}$ satisfying $B_{n}=K n^{\beta}+O\left(n^{\beta-1}\right)$ with $K \neq 0$, there exists a Dirichlet series $A$ with $\sigma_{\mathrm{c}}(A)=\alpha, A(\beta)=0$ and $\sigma_{\mathrm{c}}(A B)=\frac{1}{2}(\alpha+\beta)$. In particular, the abscissa of convergence given by Theorem 3.1 is best possible.

Proof. We can assume that $K=\beta=1$. As in Theorem 2.2, the Dirichlet series $A$ will be defined by blocks. We shall need the following technical lemmas: 
Lemma 3.3. Let $N \geq 16$ be the square of an even number. Set

$$
\begin{aligned}
\mathcal{A}=\{2 \sqrt{N} \leq i \leq N / 2 ; \exists k \in\{2, \ldots, \sqrt{N} / 2-1\}, & \\
& {[N /(i-1)]=k+1,[N / i]=k\}, } \\
\mathcal{B}=\{2 \sqrt{N} \leq j \leq N / 2 ; \exists k \in\{3, \ldots, \sqrt{N} / 2\}, & {[N / i]=k,[N /(j+1)]=k-1\} . }
\end{aligned}
$$

Then $|\mathcal{A}|=|\mathcal{B}|=\sqrt{N} / 2-2$. Moreover, if $\mathcal{A}=\left\{i_{k} ; k \in\{2, \ldots, \sqrt{N} / 2-1\}\right\}$ and $\mathcal{B}=\left\{j_{k} ; k \in\{3, \ldots, \sqrt{N} / 2\}\right\}$, where $k=\left[N / i_{k}\right]=\left[N / j_{k}\right]$, then

$$
\begin{gathered}
j_{k+1}=i_{k}-1, \\
j_{k}>i_{k}, \\
\frac{N}{i_{k}}-\frac{N}{j_{k}} \geq \frac{1}{2} .
\end{gathered}
$$

In particular, $\mathcal{A} \cap \mathcal{B}=\emptyset$.

For instance, if $N=100$, one has $\mathcal{A}=\left\{i_{2}=34 ; i_{3}=26 ; i_{4}=21\right\}$, while $\mathcal{B}=\left\{j_{3}=33 ; j_{4}=25 ; j_{5}=20\right\}$.

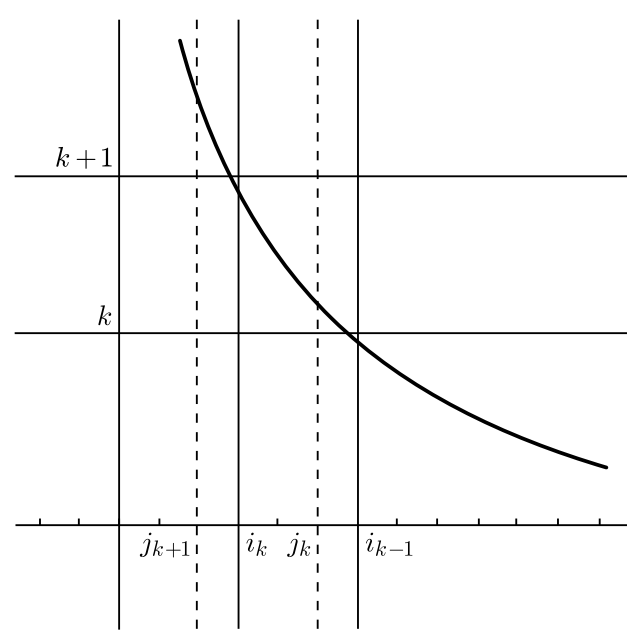

Fig. 1. Definition of the integers $i_{k}$ and $j_{k}$

Proof. First, the cardinality of each set is clearly $\leq \sqrt{N} / 2-2$. On the other hand, if $i \geq 2 \sqrt{N}$, one has:

$$
\frac{N}{i}-\frac{N}{i+1}=\frac{N}{i(i+1)} \leq \frac{1}{4}<1 .
$$

Therefore, the function $\{2 \sqrt{N}, \ldots, N / 2\} \rightarrow \mathbb{N}, i \mapsto[N / i]$, is non-increasing, and satisfies $[N /(i+1)]-[N / i] \in\{-1,0\}$. Moreover, since $[N /(2 \sqrt{N})]=$ $\sqrt{N} / 2$ and $[N /(N / 2)]=2$, each value from $\{2, \ldots, \sqrt{N} / 2-1\}$ is taken by $[N / i]$ if $i$ runs over $\{2 \sqrt{N}+1, \ldots, N / 2\}$. Fix $k$ in $\{2, \ldots, \sqrt{N} / 2-1\}$ and let $i_{k}$ 
be the first integer in $\{2 \sqrt{N}, \ldots, N / 2\}$ such that $\left[N / i_{k}\right]=k$. By definition, we have $\left[N /\left(i_{k}-1\right)\right]=k+1$, and $i_{k} \in \mathcal{A}$. This implies that $|\mathcal{A}|=\sqrt{N} / 2-2$.

The proof is exactly the same for $\mathcal{B}$. Observe that $j_{k}$ is the greatest integer in $\{2 \sqrt{N}, \ldots, N / 2\}$ with $\left[N / j_{k}\right]=k$. In particular, we get

$$
\left[\frac{N}{\left(j_{k}+1\right)-1}\right]=k, \quad\left[\frac{N}{j_{k}+1}\right]=k-1 .
$$

This shows that $j_{k}=i_{k-1}-1$.

To obtain (5), note that by construction $j_{k} \geq i_{k}$. Equality would imply

$$
\left[\frac{N}{i_{k}+1}\right]=k-1, \quad\left[\frac{N}{i_{k}-1}\right]=k+1 .
$$

But in view of the inequality $i_{k} \geq 2 \sqrt{N}+1$, this is impossible since one has

$$
\frac{N}{i_{k}-1}-\frac{N}{i_{k}+1}=\frac{2 N}{\left(i_{k}-1\right)\left(i_{k}+1\right)} \leq 1
$$

It remains to prove (6). An easy computation shows that

$$
\left(\frac{N}{j_{k}+1}-\left[\frac{N}{j_{k}+1}\right]\right)-\left(\frac{N}{j_{k}}-\left[\frac{N}{j_{k}}\right]\right)=1-\frac{N}{j_{k}\left(j_{k}+1\right)} \geq \frac{3}{4} .
$$

Since $x-[x] \in\left[0,1\left[\right.\right.$, one has $N / j_{k}-k \leq 1 / 4$. With exactly the same argument, one can prove that $N / i_{k}-k \geq 3 / 4$, which gives (6).

The following lemma is crucial.

LEMmA 3.4. Let $N \geq 16$ be the square of an even number, and let $\mathcal{A}$ and $\mathcal{B}$ be the sets defined in Lemma 3.3. For $i \in\{2 \sqrt{N}, \ldots, N / 2\}$, define a complex number $a_{i}$ as follows:

- for $k \in\{2, \ldots, \sqrt{N} / 2\}$, set $a_{i_{k}}=i_{k}^{\alpha}$, and $a_{j_{k}}=-j_{k}^{\alpha}$,

- for $i \geq 2 \sqrt{N}, i \notin \mathcal{A} \cup \mathcal{B}$, set $a_{i}=0$.

Then there exists a constant $\delta>0$, which does not depend on $N$, such that

$$
\left|\sum_{i=2 \sqrt{N}}^{N / 2}\left(\frac{N}{i}-B_{[N / i]}\right) a_{i}\right| \geq \delta N^{(1+\alpha) / 2} .
$$

Proof. For commodity reasons, we shall write $B_{n}=n+u_{n}$, where $\left(u_{n}\right)$ is a bounded sequence. Then the left hand side of (7) reads

$$
\begin{gathered}
\sum_{i=2 \sqrt{N}}^{N / 2}\left(\frac{N}{i}-B_{[N / i]}\right) a_{i}=\left(\frac{N}{i_{2}}-B_{\left[N / i_{2}\right]}\right) a_{i_{2}}+\left(\frac{N}{j_{\sqrt{N} / 2}}-B_{\left[N / j_{\sqrt{N} / 2}\right]}\right) a_{j_{\sqrt{N} / 2}} \\
\quad+\sum_{k=3}^{\sqrt{N} / 2-1} i_{k}^{\alpha}\left(\frac{N}{k}-k-u_{k}\right)-j_{k}^{\alpha}\left(\frac{N}{k}-k-u_{k}\right)=O\left(N^{\alpha}\right)+\sum_{k=3}^{\sqrt{N} / 2-1} d_{k}
\end{gathered}
$$


where

$$
d_{k}=i_{k}^{\alpha}\left(\frac{N}{i_{k}}-k-u_{k}\right)-j_{k}^{\alpha}\left(\frac{N}{j_{k}}-k-u_{k}\right) .
$$

We shall prove that

$$
d_{k} \geq \frac{1}{2} i_{k}^{\alpha}-M\left(j_{k}^{\alpha}-i_{k}^{\alpha}\right)
$$

where $M$ is a constant independent of $k$ and $N$. We consider three cases:

- If $0 \leq N / j_{k}-k-u_{k} \leq N / i_{k}-k-u_{k}$, it follows from (6) that

$$
\frac{N}{i_{k}}-k-u_{k} \geq\left(\frac{N}{j_{k}}-k-u_{k}\right)+\frac{1}{2} \geq 0
$$

which gives

$$
d_{k} \geq \frac{1}{2} i_{k}^{\alpha}-\left(\frac{N}{j_{k}}-k-u_{k}\right)\left(j_{k}^{\alpha}-i_{k}^{\alpha}\right) \geq \frac{1}{2} i_{k}^{\alpha}-M\left(j_{k}^{\alpha}-i_{k}^{\alpha}\right),
$$

where $M=1+\max \left|u_{k}\right|$.

- If $N / j_{k}-k-u_{k} \leq N / i_{k}-k-u_{k} \leq 0$, then by (6), we have

$$
-\left(\frac{N}{j_{k}}-k-u_{k}\right) \geq-\left(\frac{N}{i_{k}}-k-u_{k}\right)+\frac{1}{2} \geq 0,
$$

which gives

$$
d_{k} \geq \frac{1}{2} j_{k}^{\alpha}-\left(\frac{N}{i_{k}}-k-u_{k}\right)\left(j_{k}^{\alpha}-i_{k}^{\alpha}\right) \geq \frac{1}{2} i_{k}^{\alpha}-M\left(j_{k}^{\alpha}-i_{k}^{\alpha}\right) .
$$

- If $N / j_{k}-k-u_{k} \leq 0 \leq N / i_{k}-k-u_{k}$, we are in the most favorable case, since we add two numbers with the same sign:

$$
d_{k} \geq i_{k}^{\alpha}\left(\frac{N}{i_{k}}-k-u_{k}\right)-i_{k}^{\alpha}\left(\frac{N}{j_{k}}-k-u_{k}\right) \geq \frac{1}{2} i_{k}^{\alpha} .
$$

So, we have proved (8). Now,

$$
\sum_{k \geq 3}^{\sqrt{N} / 2-1} i_{k}^{\alpha} \geq\left(\frac{\sqrt{N}}{2}-3\right)(2 \sqrt{N})^{\alpha} \geq \delta_{1} N^{(1+\alpha) / 2} .
$$

On the other hand, we use (4) to majorize $\left|\sum_{k=3}^{\sqrt{N} / 2-1}\left(j_{k}^{\alpha}-i_{k}^{\alpha}\right)\right|$ :

$$
\begin{aligned}
\sum_{k=3}^{\sqrt{N} / 2-1}\left(j_{k}^{\alpha}-i_{k}^{\alpha}\right) & =\sum_{k=2}^{\sqrt{N} / 2-2} j_{k+1}^{\alpha}-\sum_{k=3}^{\sqrt{N} / 2-1} i_{k}^{\alpha} \\
& =O\left(N^{\alpha}\right)+\sum_{k=3}^{\sqrt{N} / 2-2}\left(\left(i_{k}-1\right)^{\alpha}-i_{k}^{\alpha}\right)
\end{aligned}
$$




$$
\begin{aligned}
& =O\left(N^{\alpha}\right)+\sum_{k=3}^{\sqrt{N} / 2-2} O\left(i_{k}^{\alpha-1}\right) \\
& =O\left(N^{\alpha}\right)+\sum_{k=3}^{\sqrt{N} / 2-2} O\left(N^{\alpha-1}\right)=O\left(N^{\alpha}\right) .
\end{aligned}
$$

Finally, we obtain

$$
\left|\sum_{i=1}^{N}\left(\frac{N}{i}-\left[\frac{N}{i}\right]-u_{[N / i]}\right) a_{i}\right| \geq \delta_{2} N^{(1+\alpha) / 2} .
$$

Proof of Theorem 3.2. We set $M_{n}=2^{4^{n}}$ for $n \geq 1$. Let $\mathcal{A}_{n}=\left\{i_{k, n}\right\}$ and $\mathcal{B}_{n}=\left\{j_{k, n}\right\}$ be the sets defined in Lemma 3.3 for $N=M_{n}$. Since $\mathcal{A}_{n} \cup \mathcal{B}_{n} \subset\left[2 \sqrt{M_{n}} ; M_{n} / 2\right]$ and $M_{n} / 2<2 \sqrt{M_{n+1}}$, the sets $\mathcal{A}_{n} \cup \mathcal{B}_{n}$ and $\mathcal{A}_{m} \cup \mathcal{B}_{m}$ are disjoint if $n \neq m$. So, we may define a sequence $\left(a_{i}\right)_{i \geq 2}$ by:

$$
a_{i_{k, n}}=i_{k, n}^{\alpha}, \quad a_{j_{k, n}}=-j_{k, n}^{\alpha}, \quad a_{i}=0 \quad \text { otherwise. }
$$

Let us verify that $\sum_{i \geq 2} a_{i} i^{-1}$ converges. It is sufficient to prove that $m \mapsto$ $\sum_{i=2}^{m} a_{i} i^{-\alpha}$ is bounded. We apply the same decomposition as in (2). Denoting by $n_{0}$ the least integer such that $N \leq M_{n_{0}}$, we have

$$
\begin{aligned}
\sum_{i=1}^{N} a_{i} i^{-\alpha} & =\sum_{n=1}^{n_{0}-1} \sum_{k=2}^{\sqrt{M_{n_{0}}} / 2-1}\left(i_{k, M_{n_{0}}}^{\alpha} i_{k, M_{n_{0}}}^{-\alpha}-j_{k, M_{n_{0}}}^{\alpha} j_{k, M_{n_{0}}}^{-\alpha}\right)+\sum_{i \geq 2 \sqrt{M_{n_{0}}}}^{N} a_{i} i^{-\alpha} \\
& =\sum_{i \geq 2 \sqrt{M_{n_{0}}}}^{N} a_{i} i^{-\alpha} .
\end{aligned}
$$

Now, properties (4) and (5) ensure that

$$
\sum_{i \geq 2 \sqrt{M_{n_{0}}}}^{N} a_{i} i^{-\alpha} \in\{-1,0,1\} .
$$

So, it is correct to set $a_{1}=-\sum_{i \geq 2} a_{i} i^{-1}$ and to consider the Dirichlet series $A(s)=\sum_{i \geq 1} a_{i} i^{-s}$. By construction, $\sigma_{\mathrm{c}}(A)=\alpha$ and $A(1)=0$. It remains to prove that $\sigma_{\mathrm{c}}(A B) \geq(1+\alpha) / 2$, since we already know that $\sigma_{\mathrm{c}}(A B) \leq(1+\alpha) / 2$. Letting $C_{N}=\sum_{i=1}^{N} a_{i} B_{[N / i]}$, this will be done by proving that

$$
\limsup _{N \rightarrow+\infty} \frac{C_{N}}{N^{(1+\alpha) / 2}}>0 .
$$

For $N=M_{n}$, we take advantage of $\sum_{i \geq 1} a_{i} i^{-1}=0$ and of the vanishing of 
$a_{i}$ for $i$ between $N^{1 / 4}$ and $2 N^{1 / 2}$ :

$$
\begin{aligned}
C_{N} & =\sum_{i=1}^{N^{1 / 4}} a_{i}\left(B_{[N / i]}-\frac{N}{i}\right)+\sum_{i=2 \sqrt{N}}^{N} a_{i}\left(B_{[N / i]}-\frac{N}{i}\right)+N \sum_{i>N} \frac{a_{i}}{i} \\
& =: S_{1}+S_{2}+S_{3} .
\end{aligned}
$$

By assumption, $B_{[N / i]}-N / i=O(1)$, which implies

$$
\left|S_{1}\right| \leq \sum_{i=1}^{N^{1 / 4}}\left|a_{i}\right|=O\left(N^{(1+\alpha) / 4}\right) .
$$

An Abel summation by parts now gives

$$
\left|S_{3}\right| \leq O\left(N^{\alpha}\right) .
$$

Finally, Lemma 3.4 gives us an estimation of $S_{2}$ :

$$
\left|S_{2}\right| \geq \delta N^{(1+\alpha) / 2}
$$

We conclude that there exists a constant $\delta^{\prime}$ such that, providing $N=M_{n}$, we have

$$
\left|C_{N}\right| \geq \delta^{\prime} N^{(1+\alpha) / 2} .
$$

This ends to prove that $\sigma_{\mathrm{c}}(A B) \geq(1+\alpha) / 2$.

REMARK 3.5. As in [2], it is possible to give a topological proof of Theorem 3.2, which is maybe a little bit less technical. Indeed, consider a non-decreasing sequence $\left(\varphi_{N}\right)$ such that, for all Dirichlet series $A$ with $\sigma_{\mathrm{c}}(A)=\alpha$ and $A(1)=0$, the sequence $\left(c_{1}+\ldots+c_{N}\right) / \varphi_{N}$ is bounded, where $\sum_{n=1}^{+\infty} c_{n} n^{-s}=A(s) B(s)$. It is sufficient to prove that

$$
\varphi_{N} \geq \delta N^{(1+\alpha) / 2}, \quad \text { where } \delta \text { is a positive constant. }
$$

We introduce the Banach space $E=\left\{a=\left(a_{n}\right) ; \sum_{n \geq 1} a_{n} n^{-\alpha}\right.$ converges, $\left.\sum_{n \geq 1} a_{n} n^{-1}=0\right\}$, equipped with the norm $\|a\|=\sup _{n}\left|\sum_{k=1}^{n} a_{k} k^{-\alpha}\right|$. Define a sequence of linear forms $\left(L_{n}\right)$ on $E$ by

$$
L_{n}(a)=\frac{c_{1}+\ldots+c_{n}}{\varphi_{n}}
$$

By our assumption, $\sup _{n}\left|L_{n}(a)\right|<\infty$ for each $a \in E$. The Banach-Steinhaus theorem now gives $M=\sup _{n}\left\|L_{n}\right\|<+\infty$, i.e. for each $a \in E$ and each $n \in \mathbb{N}^{*}$,

$$
\left|\sum_{i=1}^{n} a_{i} B_{[n / i]}\right| \leq M \varphi_{n}\|a\| .
$$

Suppose that $N$ is the square of an even number; again, $\mathcal{A}$ and $\mathcal{B}$ are defined in Lemma 3.3, and the sequence $\left(a_{i}\right)$ is defined as follows:

- For $k \in\{2, \ldots, \sqrt{N} / 2\}$, we set $a_{i_{k}}=i_{k}^{\alpha}$ and $a_{j_{k}}=-j_{k}^{\alpha}$.

- For $i \geq 2, i \notin \mathcal{A} \cup \mathcal{B}$, we set $a_{i}=0$. 
- Finally, we set $a_{1}=-\sum_{i=2}^{N} a_{i} i^{-1}$.

This definition is consistent, and $a \in E$. Now, the computation made in the proof of Theorem 3.2 implies that $\|a\|=O(1)$ and

$$
\left|\sum_{i=1}^{N} a_{i} B_{[N / i]}\right| \geq \delta N^{(1+\alpha) / 2} .
$$

This in turn implies the required inequality on $\varphi_{N}$.

REMARK 3.6. Conditions 1 and 2 in Theorem 3.1 cannot be dispensed with.

1. If we do not assume that $A(\beta)=0$, the conclusion is false. For example, if $0<\alpha<1$, consider $B(s)=\zeta(s)$ and $A(s)=\zeta(s-1+\alpha)$. Then

$$
\sum_{i=1}^{n} a_{i} B_{[n / i]} \geq \sum_{i=1}^{n} a_{i} \frac{n}{i}-\sum_{i=1}^{n} a_{i} \geq n \sum_{i=1}^{n} \frac{1}{i^{2-\alpha}}-\sum_{i=1}^{n} \frac{1}{i^{1-\alpha}} \geq \delta n
$$

which proves that the abscissa of convergence is at least 1 , while Theorem 3.1 would give $\frac{1}{2}(1+\alpha)$.

2. Now consider a Dirichlet series $B$ such that $B_{n}=n+(-1)^{n} n^{r}, 0<$ $r<1$. If $N$ is the square of an even integer, and the set $\mathcal{A}$ is defined as previously, we choose $a \in E$ with $a_{i_{k}}=(-1)^{k} i_{k}^{\alpha}, a_{i}=0$ for $i \geq 2, i \neq i_{k}$, and $a_{1}=-\sum_{i \geq 2} a_{i} i^{-1}$. It is clear that $a \in E$, and that $\|\bar{a}\|=O(1)$. Moreover

$$
\begin{aligned}
\sum_{i=1}^{N} a_{i} B_{[N / i]} & =\sum_{i=1}^{N} a_{i}\left(B_{[N / i]}-\frac{N}{i}\right) \\
& =\sum_{k=2}^{\sqrt{N} / 2-1} a_{i_{k}}\left(k-\frac{N}{i_{k}}\right)+\sum_{k=2}^{\sqrt{N} / 2-1} a_{i_{k}}(-1)^{k} k^{r} \\
& =O\left(\sum_{k=2}^{\sqrt{N} / 2-1}\left|a_{i_{k}}\right|\right)+\sum_{k=2}^{\sqrt{N} / 2-1} i_{k}^{\alpha} k^{r}=O\left(N^{(1+\alpha) / 2}\right)+\sum_{k=2}^{\sqrt{N} / 2-1} i_{k}^{\alpha} k^{r}
\end{aligned}
$$

But $i_{k} \geq \sqrt{N}$, and so

$$
\sum_{k=2}^{\sqrt{N} / 2-1} i_{k}^{\alpha} k^{r} \geq C N^{\alpha / 2} N^{(1+r) / 2} \geq C N^{(1+\alpha) / 2+r / 2} .
$$

This gives $\varphi_{N} \geq \delta N^{(1+\alpha) / 2+r / 2}$, which means that we cannot improve the abscissa of convergence given by Theorem 1.1.

Acknowledgements. We thank M. Balazard for stimulating conversations. 


\section{References}

[1] H. Bohr, On the summability functions and order functions of Dirichlet series, Dan. Mat. Fys. Medd. 27 (1952), 3-38 (manuscript prepared by E. Folner) (I A 22 in Collected Math. Works).

[2] J. P. Kahane et H. Queffélec, Ordre, convergence et sommabilité des produits de séries de Dirichlet, Ann. Inst. Fourier (Grenoble) 47 (1997), 485-529.

[3] S. V. Konyagin and H. Queffélec, The translation $1 / 2$ in the theory of Dirichlet series, Real Anal. Exchange 27 (2001/2002), 155-175.

[4] E. Landau, Über die Multiplikation Dirichlet'scher Reihen, Rend. Circ. Mat. Palermo 25 (1907), 81-160.

[5] H. Queffélec, Propriétés presque sûres et quasi sûres des séries de Dirichlet et des produits d'Euler, Canad. J. Math. 32 (1980), 531-558.

[6] G. Tenenbaum, Introduction à la théorie analytique et probabiliste des nombres, Cours spécialisé de la SMF, 1995.

Laboratoire AGAT

Université des Sciences et Technologies de Lille

Bât. M2

F-59655 Villeneuve d'Ascq Cedex, France

E-mail: bayart@agat.univ-lille1.fr
Current address:

LabaG

Université Bordeaux I

351, Cours de la Libération

F-33405 Talence Cedex, France

E-mail: bayart@math.u-bordeaux.fr

Received on 29.4.2002

and in revised form on 10.2.2003 